overestimated daily exposure and exposure during application. However exposure was underestimated at mixing/loading in many observations, especially when the operator wore long working clothes or gloves.

Conclusion The AOEM model did not appear conservative in the sense that it did not overestimate exposures in all circumstances. More specifically: 1) the overestimation at spraying appeared a consequence of the overestimation of daily treated area, 2) the protection provided by PPE appeared overestimated, 3) mixing/loading exposure, a phase in which operators are exposed to concentrated products, appeared underestimated. These discrepancies could be due to optimal working conditions (larger farms, newer equipment) under which industries' studies are conducted that are not representative of operators' actual working conditions in fruit growing.

\section{0-185 ASSESSING OCCUPATIONAL AND ENVIRONMENTAL DEPLOYMENT-RELATED MILITARY EXPOSURE AMONG U.S. VETERANS}

1Paul D Blanc, Anna Korpak, Andrew Timmons, Karen Nakayama, Susan Proctor, Nicholas Smith, Eric Garshik. 'University of California San Francisco, United States

\subsection{6/OEM-2021-EPI.47}

Introduction Twenty-first century occupational and environmental (OE) exposures arising from military service encompass combustion byproducts, particulate matter, and traditional job exposures.

Rationale Multiple potentially collinear exposures can require data reduction to facilitate epidemiological analyses. Our data provided an opportunity to characterize relationships among a range of interrelated exposures.

Methods We analyzed interim data from the Veterans Affairs 'CSP \#595: Service and Health Among Deployed Veterans' study. As of May 2020, survey responses were available from 1962 randomly selected Veterans with one or more deployments to Afghanistan, Iraq, or elsewhere in southwest Asia between 2001 and 2017. The interviewer-administered questionnaire yielded 3-level responses to 32 OE deployment-associated exposures included in this analysis. We identified a priori six anticipated exposure factors: burn pit (4 items); other open combustion sources (5 items); combustion engine byproducts (5 items); mechanically generated dust/dust storms (4 items); occupational vapors, gas, dust, and fumes (VGDF; 11 items); and other toxicants (3 items). We used confirmatory factor analysis (CFA) to assess construct validity of these groupings.

Results In preliminary CFA, two of six groupings that were highly collinear (combustion engine byproducts/mechanically generated dust) required combination for model viability. The resulting 5 factor model performed adequately (incremental or Comparative Fit Index $[\mathrm{CFI}]=0.945)$. After excluding 4 of 32 items with lower factor loadings ( 0.50 for 28 3-level items on five factors.

Conclusion These findings provide a basis for item reduction in an expansive survey battery of exposure items addressing occupational and environmental military exposures. We identified 28 items (three response levels) comprising five distinct factors. These results suggest that among previously deployed Veterans, multiple OE exposures can be simplified to exposure-related factors as part of assessing health effects.

\section{0-262 IMPACT OF OCCUPATIONAL PESTICIDE EXPOSURE ASSESSMENT METHODS ON RISK ESTIMATES FOR PROSTATE CANCER, NON-HODGKIN'S LYMPHOMA AND PARKINSON'S DISEASE - RESULTS OF THREE META- ANALYSES}

'Johan Ohlander, Samuel Fuhrimann, Martie van Tongeren, Roel Vermeulen, Anke Huss, Hans Kromhout. 'Utrecht University, Netherlands

\subsection{6/OEM-2021-EPI.48}

Introduction Assessment of occupational pesticide exposure in epidemiological studies of chronic diseases is particularly challenging. Biomonitoring of current pesticide levels might not correlate with past exposure relevant to disease etiology, and indirect methods often rely on workers' imperfect recall of exposures, or workers' job titles.

\section{Objectives}

Within the IMPRESS project (www impress-project.org) we investigated how exposure assessment method (EAM) type for assessing occupational pesticide exposure influenced risk estimates for some chronic diseases.

Methods In three meta-analyses the influence of type of EAM on the pooled risk of prostate cancer (25 articles), Non-Hodgkin's lymphoma (NHL) (29 articles), and Parkinson's disease (PD) (34 articles) was investigated using subgroup analyses by type of EAM. Data were mainly obtained from a previous systematic review conducted by the authors. Categories of EAM types used were: group-level assessments (e.g. job titles), self-reported exposures, expert-level assessments (e.g. job-exposure matrices), and biomonitoring. Further sub-group analyses were made by study design and publication year.

Results EAM types were not associated with statistically significantly different pooled risk estimates regarding any health outcome. However, for all health outcomes, case-control studies showed consistently higher risk estimates when expert-level assessments were used compared with self-reports. Overall, case-control designs showed higher risk estimates than cohort designs. Cancer studies showed higher risk estimates in later publications, whereas PD studies showed higher risk estimates in earlier publications.

Conclusion Overall, EAM type in studies of occupational pesticide exposure appears not to affect risk estimates of prostate cancer, NHL, and PD. Nevertheless, in case-control settings self-reported exposures might yield lower risk estimates than expert-based methods, possibly resulting from a higher degree of exposure misclassification due to workers imperfect recall of exposures. In systematic reviews of health effects of occupational exposure to pesticides, study design, year of publication, and exposure assessment method(s) should be taken into account. 